\title{
Publisher Correction: Experimental demonstration of a three-dimensional lithium niobate nonlinear photonic crystal
}

Dunzhao Wei, Chaowei Wang, Huijun Wang, Xiaopeng Hu, Dan Wei, Xinyuan Fang, Yong Zhang (D), Dong Wu, Yanlei Hu, Jiawen Li, Shining Zhu and Min Xiao

Correction to: Nature Photonics https://doi.org/10.1038/s41566-018-0240-2, published online 20 August 2018.

In the HTML version of this Letter originally published, Yong Zhang, Shining Zhu and Min Xiao weren't shown as corresponding authors; this has now been corrected. The PDF version was unaffected.

Published online: 9 September 2020

https://doi.org/10.1038/s41566-020-00698-3

(๑) The Author(s), under exclusive licence to Springer Nature Limited 2020 